\title{
Effects of intraspecific competition of prey in the dynamics of a food chain model
}

\author{
Pinki Shome $^{1} \cdot$ Alakes Maiti $^{2} \cdot$ Swarup Poria $^{1}$
}

Received: 6 September 2016/ Accepted: 11 October 2016/Published online: 27 October 2016

(C) Springer International Publishing Switzerland 2016

\begin{abstract}
In this paper, dynamical behavior of simple prey-predator model with Holling type II functional response involving additional food for predator along with intraspecific competition of prey is proposed and analyzed. The stability criteria of solutions are investigated by varying quantity and quality of additional food and intraspecific competition of prey population. Conditions for Hopf bifurcation are derived analytically. Numerical simulation results are presented to observe the time evolution of the system. This study may be useful to understand the effects of intraspecific competition in real world ecological systems.
\end{abstract}

Keywords Prey-predator model - Additional food . Intraspecific competition $\cdot$ Stability analysis $\cdot$ Hopf bifurcations

\section{Introduction}

In light of the conservation of biodiversity, it is of paramount importance to understand the effects of variation of predator population in an ecosystem. During the last few

Pinki Shome

pinkishome@yahoo.co.in

Alakes Maiti

alakesh_maity@hotmail.com

Swarup Poria

swarup_p@yahoo.com

1 Department of Applied Mathematics, University of Calcutta, 92 APC Road, Kolkata 700009, India

2 Department of Mathematics, Vidyasagar Evening College, 39, Sankar Ghosh Lane, Kolkata 700006, India decades continuous attention is given to prey-predator model for its wide range of applications in mathematical biology. Additional foods are important components of most predators (e.g., coccinellid) diet, although they receive less attention than basal prey in the scientific literature, these foods fundamentally shape the life histories of many predator species (cf. Sahoo and Poria 2014). In recent years, many biologist, experimentalists and theoreticians investigated the consequences of providing additional food to predators in a predator prey systems (cf. Kumar and Freedman 1989; Sahoo and Poria 2013; Sabelis and Rijn 2005; Gakkhar and Singh 2012). Along with additional food's quality and quantity, intraspecific competition of prey population can influence the change in stability of prey-predator system. Intraspecific competition mainly reduces population growth rates when population density used to increase. Intraspecific competition doesn't have any impact on population when biological resources are seems to be infinite. This phenomenon leads exponential population growth which is exceedingly rare in nature. Introduction of intraspecific competition makes prey-predator interactions more realistic. In recent years prey-predator system dynamics are investigated by Pitchford and Brindley (cf. Pitchford and Brindley 1998) incorporating the effects of intraspecific competition.

One of the most important factors of mathematical models of prey-predator system is the functional response (cf. Agiza et al. 2009; Yujing 2013). After the introduction of Holling (cf. Holling 1959a, b, 1965) many types of functional responses (cf. Ruan and Xiao 2001; Saeez and Gonzalez-Olivares 1999; Skalski and Gilliam 2001; Akcakaya et al. 1995; Wang et al. 2007) are used among which Holling type II, III and IV are the extensively used in literature of mathematical biology (cf. Aziz-Alaoui and Okiye 2003; Camara and Alaoui 2008; Mukherjee et al. 
2011; Lu and Wang 2011; Zhang et al. 2008; Agarwal and Pathak 2012; Chen et al. 2013; Zhen and Zhong 2013). The effects of the presence of additional food in the ecological system is taken into account through the modification of functional response term (cf. Sahoo and Poria 2011, 2013, 2015; Srinivasu and Prasad 2010). In real world ecology when population used to approach carrying capacity reproduction rate and survival process usually decrease due to intraspecific competition. Intraspecific competition generally occurs due to competition for searching mates, resources which can influence change in structure of species population as well as life history of species. There are many theoretical studies (cf. Pal et al. 2009; Chakraborty et al. 2015; Sabelis and Rijn 2006; Sahoo and Poria 2013, 2014; Srinivasu and Prasad 2011; Prasad et al. 2013) incorporating additional food to predator only assuming logistic growth of prey. However, none of the model has investigated the effects of variation of growth rate of prey population due to different kind of intra-specific competitions among prey populations. These facts motivate us to investigate the effects of variation of growth rate of prey population in a predator prey system in presence of additional food for predator in this paper. In particular we consider the $\theta$ logistic growth of prey population and study the effect of variation of $\theta$ (cf. Ross 2009). Notice that for $\theta=1$ we have the usual logistic growth rate of prey which was used in the previous investigations made by Srinivasu (cf. Srinivasu et al. 2007).

The section wise split of the paper are the following. In "Model", the model is discussed and in "Analysis of model" the stability of the model is analyzed. In "Simulation results" simulation results of the paper are discussed and in "Conclusions" conclusions are drawn.

\section{Model}

We consider $\theta$-logistic growth of prey and formulate the following predator-prey model

$$
\begin{aligned}
& \frac{d N}{d T}=r N\left(1-\left(\frac{N}{K}\right)^{\theta}\right)-\frac{C N P}{a+N}, N(0)>0 \\
& \frac{d P}{d T}=\frac{b N P}{a+N}-m P, P(0)>0 .
\end{aligned}
$$

where prey population $N$ has carrying capacity $K>0$ and intrinsic growth rate $r>0, m$ is death rate of predator $P$ as well as starvation rate. We consider $h_{1}$ as handling time of predator per prey item and $e_{1}$ as ability of the predator to detect the prey, then $C=\frac{1}{h_{1}}$ represents maximum rate of predation by predator and $a=\frac{1}{e_{1} h_{1}}$ is half saturation value of predator. If the efficiency with which the food consumed by the predator used to be converted into predator biomass is $\epsilon$ then $b=\frac{\epsilon}{h_{1}}$ is the maximum growth rate of the predator. Now it is assumed that predator is provided with additional food having biomass $A$ which is assumed to be distributed uniformly in the habitat. It is assumed that the number of encounters per predator with the additional food is proportional to the density of the additional food. The proportionality constant characterizes the ability of the predator to identify the additional food. Thus when additional food is supplied to predator then the previous system will be of the following form

$$
\begin{aligned}
& \frac{d N}{d T}=r N\left(1-\left(\frac{N}{K}\right)^{\theta}\right)-\frac{C N P}{a+\alpha \eta A+N}, N(0)>0 \\
& \frac{d P}{d T}=\frac{b(N+\eta A) P}{a+\alpha \eta A+N}-m P, P(0)>0 .
\end{aligned}
$$

Handling time of predator per unit quantity of additional food is represented by $h_{2}$ and if ability for the predator to detect the additional food is $e_{2}$ then $\eta=\frac{e_{2}}{e_{1}}$ and $\alpha=\frac{h_{2}}{h_{1}}$. The term $\eta A$ here represents effectual additional food level. System (2) reduces to system (1) when $A=0$. We will analyze the system (2) for studying its controllability with respect to the quantity and quality of additional food. We non-dimensionalize the system (1) and (2) using transformations

$x=\frac{N}{a}, y=\frac{C P}{a r}$, and $t=r T$.

Then the system (2) takes the form

$$
\begin{aligned}
& \frac{d x}{d t}=x\left(1-\frac{x^{\theta}}{\gamma^{\theta}}\right)-\frac{x y}{1+\alpha \xi+x}, x(0)>0 \\
& \frac{d y}{d t}=\frac{\beta x y}{1+\alpha \xi+x}-\delta y, y(0)>0 .
\end{aligned}
$$

where $\gamma=\frac{K}{A}, \beta=\frac{b}{r}, \delta=\frac{m}{r}, \xi=\frac{\eta A}{a}$.

$$
\begin{aligned}
\text { We define } f(x) & =\frac{x}{1+\alpha \xi+x}, \\
\text { and } g(x) & =(1+\alpha \xi+x)\left(1-\frac{x^{\theta}}{\gamma^{\theta}}\right) .
\end{aligned}
$$

The system (3) becomes

$$
\begin{aligned}
& \frac{d x}{d t}=f(x)[g(x)-y], x(0)>0 \\
& \frac{d y}{d t}=\left[\beta f(x)\left(1+\frac{\xi}{x}\right)-\delta\right] y, y(0)>0 .
\end{aligned}
$$

Transformed system of (1) will be obtained when $\xi=0$. 


\section{Analysis of model}

\section{Boundedness of solution}

In this section, we shall show first that all solutions of the model with non-negative initial conditions will remain bounded.

Theorem All solution of system (4) that start in $R_{+}^{2}$ are uniformly bounded.

Proof

We take

$$
\begin{aligned}
W(t) & =x(t)+y(t) \\
W^{\prime}(t) & =\frac{d x}{d t}+\frac{d y}{d t} \\
& =x\left(1-\frac{x^{\theta}}{\gamma^{\theta}}\right)-\frac{x y}{1+\alpha \xi+x}+\frac{\beta(x+\xi) y}{1+\alpha \xi+x}-y \delta \\
& \leq x\left(1-\frac{x^{\theta}}{\gamma^{\theta}}\right)-y \delta \\
& \leq 2 x-y \delta-x \\
& \leq 2-\lambda(x+y)
\end{aligned}
$$

Therefore

$$
\begin{aligned}
& \frac{d W}{d t}+W \lambda \leq 2 \\
& \quad \text { hence } 0<W<\frac{2\left(1-e^{-t \lambda}\right)}{\lambda}+W(x(0), y(0)) e^{-t \lambda}
\end{aligned}
$$

here $\lambda=\min \{1, \delta\}$. For $t \rightarrow \infty$ we have $0<W<\frac{2}{\lambda}$, hence $0<W<M$, where $M=\frac{2}{\lambda}$. Hence all the solutions of the system (4) initiating in $R_{+}^{2}$ are confined in the region $S_{1}=$ $(x, y) \in R_{+}^{2}: W=\frac{2}{\lambda}+\zeta$ for any $\zeta>0$, which means all species are uniformly bounded for any initial value in $R_{+}^{2}$. This proves the theorem.

\section{Equilibria and their stability conditions}

Now we determine the equilibrium points of the system and discuss their stability conditions when $\xi=0$ and draw conclusion regarding the behavior of stability of equilibrium. Prey isocline of system (4) is $y=(1+\alpha \xi+x)$ $\left(1-\frac{x^{\theta}}{\gamma^{\theta}}\right)$ which is an increasing function of both $\alpha$ and $\xi$ in $[0, \gamma]$ which intersect $\mathrm{y}$ axis at $(0,1+\alpha \xi)$ and $\mathrm{x}$ axis at $(\gamma, 0)$. Predator isocline of system (4) is $x=\frac{\delta(1+\alpha \xi)-\beta \xi}{\beta-\delta}$ which is a straight line. Prey isocline of system when $\xi=0$ is $y=(1+x)\left(1-\frac{x^{\theta}}{\gamma^{\theta}}\right)$, predator isocline of system when $\xi=0$ is $x=\frac{\delta}{\beta-\delta}$. The predator isocline of system (4) may move to the right or left from $x=\frac{\delta}{\beta-\delta}$ as $\xi$ increases from zero depending on the relative position of $\alpha$ with respect to $\frac{\beta}{\delta}$. The equilibrium point of system (4) is

$$
\left(x^{*}, y^{*}\right)=\left(\frac{\delta+\xi(\alpha \delta-\beta)}{\beta-\delta}, g\left(x^{*}\right)\right)
$$

and for $\xi=0$ the equilibrium point is

$$
(\tilde{x}, \tilde{y})=\left(\frac{\delta}{\beta-\delta}, g(\tilde{x})\right)
$$

Clearly it is observed that $y^{*}>\tilde{y}$ when $\xi>0$. If $\alpha<\frac{\beta}{\delta}$ then $x^{*}<\tilde{x}$ and if $\alpha>\frac{\beta}{\delta}$ then $x^{*}>\tilde{x}$. As $x^{*} \geq 0$ so $\xi \geq \frac{\delta}{\beta-\delta \alpha}$ and from $y^{*}>0$ we get $\gamma>x^{*}$ which gives $\xi>\frac{\delta-\gamma(\beta-\delta)}{\beta-\alpha \delta}$. Hence $\alpha$ always enhances equilibrium level of predator of system (4) when $\xi>0$. If $\alpha>\frac{\beta}{\delta}$ and $\xi=0$ then if system does not admit interior equilibrium then (4) shall never admit interior equilibrium. If $\alpha<\frac{\beta}{\delta}$ then system (4) admits interior equilibrium even if when $\xi=0$ does not provide interior equilibrium, provided we have $\xi>\frac{\delta-\gamma(\beta-\delta)}{\beta-\alpha \delta}$ from $y^{*}>0$, and it will maintain the interior equilibrium if $\xi \geq \frac{\delta}{\beta-\delta \alpha}$ from $x^{*} \geq 0$. When $\alpha=\frac{\beta}{\delta}$ then $\xi=0$ gives interior equilibrium point, from system (4) the equilibrium predator population increases with additional food but the equilibrium prey population remains at the same level as that of when $\xi=0$.

Jacobian matrix of system (4) is

$$
J_{(x, y)}=\left(\begin{array}{cc}
g^{\prime}(x) f(x)+[g(x)-y] f^{\prime}(x) & -f(x) \\
\beta y\left[f^{\prime}(x)\left(1+\frac{\xi}{x}\right)-\frac{f(x) \xi}{x^{2}}\right] & \beta f(x)\left(1+\frac{\xi}{x}\right)-\delta
\end{array}\right) .
$$

Now $J_{(0,0)}=\left(\begin{array}{cc}1 & 0 \\ 0 & \frac{\beta \xi}{1+\alpha \xi}-\delta\end{array}\right)$ and

$$
J_{(\gamma, 0)}=\left(\begin{array}{cc}
-1 & -\gamma \\
0 & \beta\left(\frac{\gamma+\xi}{1+\alpha \xi+\gamma}\right)-\delta
\end{array}\right) \text {. }
$$

Here $(0,0)$ and $(\gamma, 0)$ are common equilibrium point between system having $\xi=0$ and (4).

Here $J_{\left(x^{*}, y^{*}\right)}=\left(\begin{array}{cc}g^{\prime}\left(x^{*}\right) f\left(x^{*}\right) & -f\left(x^{*}\right) \\ \beta g\left(x^{*}\right)\left[f^{\prime}\left(x^{*}\right)\left(1+\frac{\xi}{x^{*}}\right)-\frac{f\left(x^{*}\right) \xi}{x^{* 2}}\right] & 0\end{array}\right)$

where $f^{\prime}(x)=\frac{1+\alpha \xi}{(1+\alpha \xi+x)^{2}}>0$

and $g^{\prime}\left(x^{*}\right)=1-\frac{x^{\theta}}{\gamma^{\theta}}-\frac{(1+\alpha \xi+x)}{\gamma^{\theta}} \theta x^{\theta-1}$.

The dynamical behavior of system (4) is analyzed under the condition of existence and stability criteria for interior equilibrium point when $\xi=0$. The conditions are as follows 
(a)

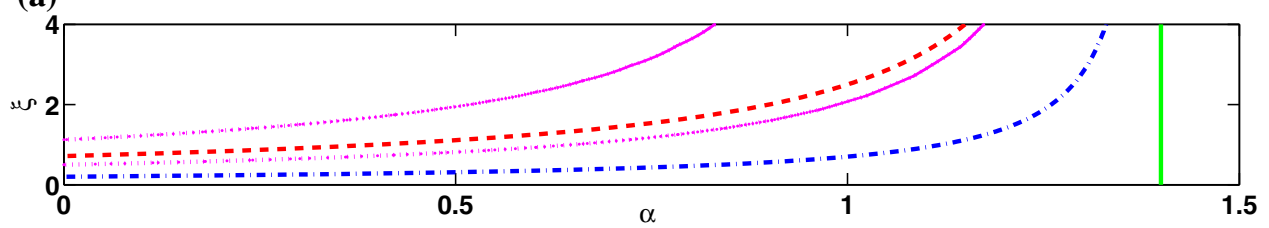

(b)

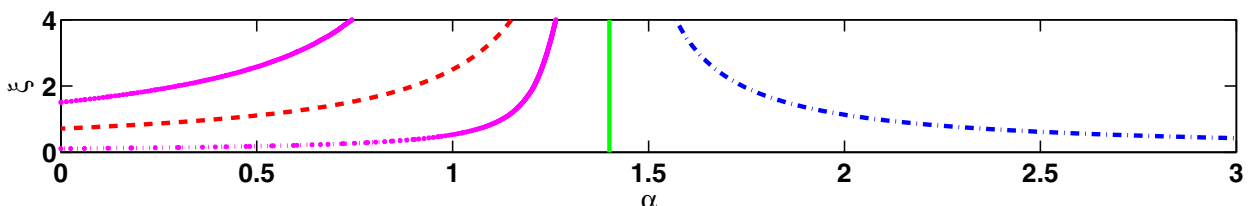

(c)

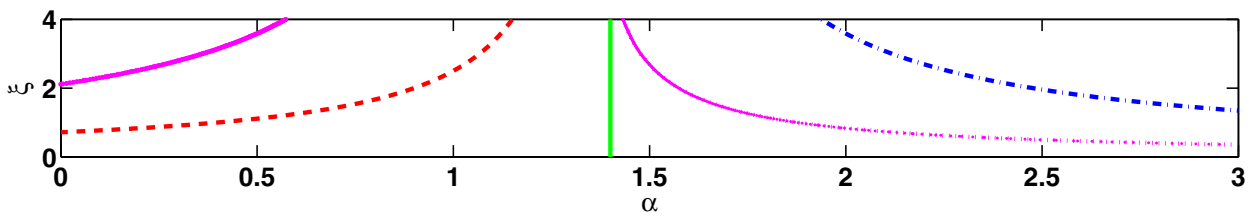

Fig. 1 Diagram for curves (10), (11), (12) under conditions (7), (8), (9) when parameter values are $\beta=0.35, \delta=0.25, \theta=2$. Dashed line represents curve equation (10), dash dotted line represents curve equation (11), dotted line represents curve equation (12) and continuous line represents the straight line $\alpha=\frac{\beta}{\delta}$. a for $\gamma=1.8$ [(following condition (7) (for fixed $\alpha$ and increasing value of $\xi$ in between 2 magenta colored curve branch system is stable when values of $\xi$ lies over the upper branch $)$ ], b for $\gamma=4.2$ [(following

$\gamma^{\theta} \leq\left(\frac{\delta}{\beta-\delta}\right)^{\theta}<\frac{\beta}{\delta}\left(\frac{\delta}{\beta-\delta}\right)^{\theta}\left(\frac{\delta}{\beta}+\theta\right)$

$\gamma^{\theta} \leq\left(\frac{\delta}{\beta-\delta}\right)^{\theta}<\frac{\beta}{\delta}\left(\frac{\delta}{\beta-\delta}\right)^{\theta}\left(\frac{\delta}{\beta}+\theta\right)$

$\left(\frac{\delta}{\beta-\delta}\right)^{\theta}<\frac{\beta}{\delta}\left(\frac{\delta}{\beta-\delta}\right)^{\theta}\left(\frac{\delta}{\beta}+\theta\right) \leq \gamma^{\theta}$

System (4) admits interior equilibrium if $\gamma>\frac{\delta}{\beta-\delta}$ and this interior equilibrium is unstable if $\gamma>\left(\frac{\delta}{\beta-\delta}\right)\left[\frac{\beta}{\delta}\left(\frac{\delta}{\beta}+\theta\right)\right]^{\frac{1}{\theta}}$, and asymptotically stable if $\gamma<\left(\frac{\delta}{\beta-\delta}\right)\left[\frac{\beta}{\delta}\left(\frac{\delta}{\beta}+\theta\right)\right]^{\frac{1}{\theta}}$.

\section{Conditions for Hopf bifurcation}

According to the stability conditions of system (4) we can conclude that Hopf bifurcation occurs at $\gamma=\left(\frac{\delta}{\beta-\delta}\right)\left[\frac{\beta}{\delta}\left(\frac{\delta}{\beta}+\right.\right.$ $\theta)]^{\frac{1}{\theta}}$ when $\xi=0$. The eigen value $\frac{\beta \xi}{1+\alpha \xi}-\delta$ of $J_{(0,0)}$ decides nature of $(0,0)$, eigen value $\frac{\beta(\gamma+\xi)}{1+\alpha \xi+\gamma}-\delta$ decides nature of $(\gamma, 0)$, and $g^{\prime}\left(x^{*}\right)$ (provides sign of $\left.J_{\left(x^{*}, y^{*}\right)}\right)$ decides nature of $\left(x^{*}, y^{*}\right)$ of the system (4). Hence in $(\alpha, \xi)$ space we will draw the curves

$\beta \xi-\delta(1+\alpha \xi)=0$ condition (8) (for fixed $\alpha$ and increasing value of $\xi$ in between 2 curve magenta colored branch system is stable when values of $\xi$ lies over the upper branch )], $\mathbf{c}$ for $\gamma=7.88$ [following condition (9) (for fixed $\alpha$ and increasing value of $\xi$ in between 2 magenta colored curve branch system is stable when values of $\xi$ lies over the upper branch when $\alpha<1.4$ and for $\alpha>1.4$ system is stable when $\xi$ lies under the magenta colored curve branch )] respectively are plotted in $(\alpha, \xi)$ space

$$
\begin{aligned}
& \beta(\gamma+\xi)-\delta(1+\alpha \xi+\gamma)=0 \\
& (\beta-\delta)^{\theta} \gamma^{\theta}-(1+\theta)[\delta+\xi(\alpha \delta-\beta)]^{\theta} \\
& \quad-\theta(1+\alpha \xi)(\beta-\delta)[\delta+\xi(\alpha \delta-\beta)]^{\theta-1}=0 .
\end{aligned}
$$

Since $\alpha=\frac{\beta}{\delta}$ is an asymptote for both (10) and (11) the dynamical behavior of system (4) is examined through the curves (10-12). The curves for $\theta=2$ are represented in Fig. 1, same for $\theta=3$ and $\theta=4$ are presented in Figs. 2, 3 respectively.

When $\alpha=\frac{\beta}{\delta}$ then system (4) will be globally asymptotically stable for $\xi>\frac{(\beta-\delta)^{\theta} \gamma^{\theta}-(1+\theta) \delta^{\theta}-\theta(\beta-\delta) \delta^{\theta-1}}{\theta \beta(\beta-\delta) \delta^{\theta-2}}$ and unstable for $\xi \in\left[0, \frac{(\beta-\delta)^{\theta} \gamma^{\theta}-(1+\theta) \delta^{\theta}-\theta(\beta-\delta) \delta^{\theta-1}}{\theta \beta(\beta-\delta) \delta^{\theta-2}}\right]$.

Hence Hopf bifurcation of interior equilibrium $\left(x^{*}, y^{*}\right)$ occurs at

$$
\begin{aligned}
& \xi=\frac{(\beta-\delta)^{\theta} \gamma^{\theta}-(1+\theta) \delta^{\theta}-\theta(\beta-\delta) \delta^{\theta-1}}{\theta \beta(\beta-\delta) \delta^{\theta-2}} \\
& \text { If } g^{\prime}\left(x^{*}\right)=1-\frac{(1+\alpha \xi) \theta\left(x^{*}\right)^{\theta-1}}{\gamma^{\theta}}-\frac{(1+\theta)}{\gamma^{\theta}}\left(x^{*}\right)^{\theta}<0
\end{aligned}
$$

then interior equilibrium of system (4) is globally asymptotically stable. From system (4) at $\left(x^{*}, y^{*}\right)$ we get 
Fig. 2 Diagram for curves (10), (11), (12) under conditions (7), (8), (9) when $\beta=0.35, \delta=$ $0.25, \theta=3$. Dashed line represents curve equation (10), dash dotted line represents curve equation (11), dotted line represents curve equation (12) and continuous line represents the straight line $\alpha=\frac{\beta}{\delta}$. a for $\gamma=2.1$ [following condition (7) (for fixed $\alpha$ and increasing value of $\xi$ in between 2 magenta colored curve branch system is stable when values of $\xi$ lies over the upper branch )], $\mathbf{b}$ for $\gamma=$ 3.9 [following condition (8)] (c) for $\gamma=6.5$ [following condition (9)] respectively are plotted in $(\alpha, \xi)$ space (a)

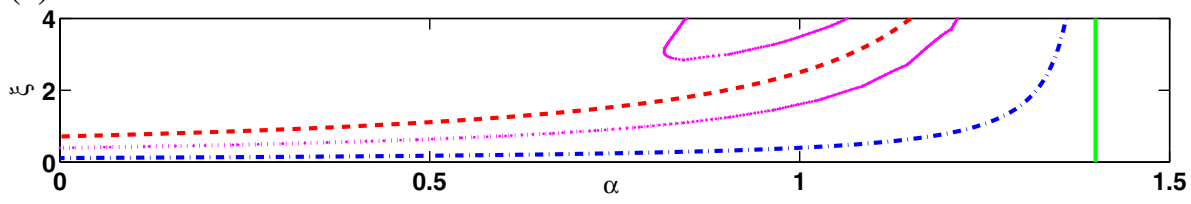

(b)

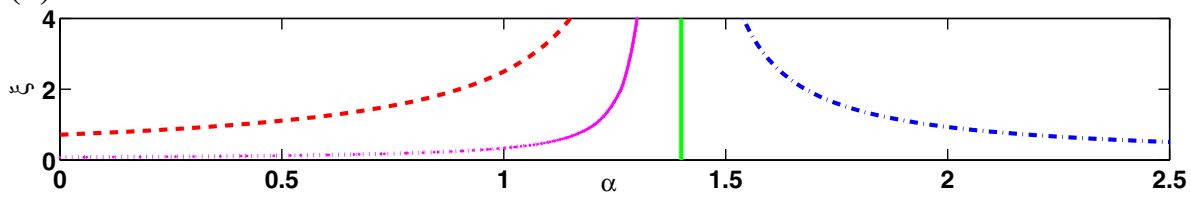

(c)

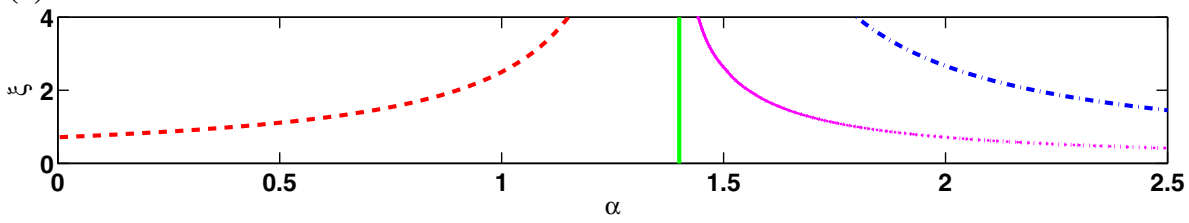

(a)

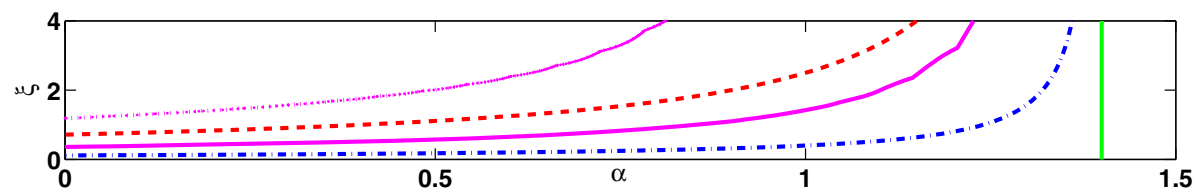

(b)

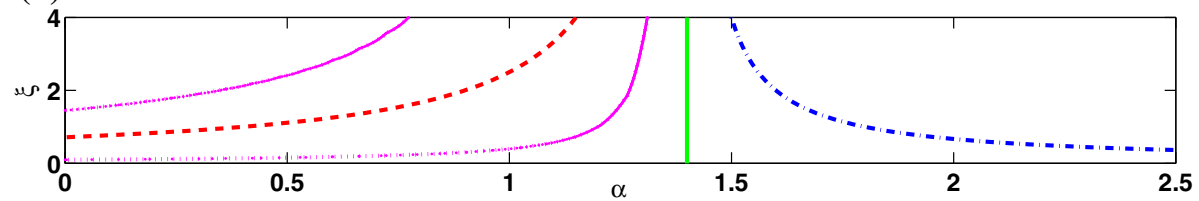

(c)

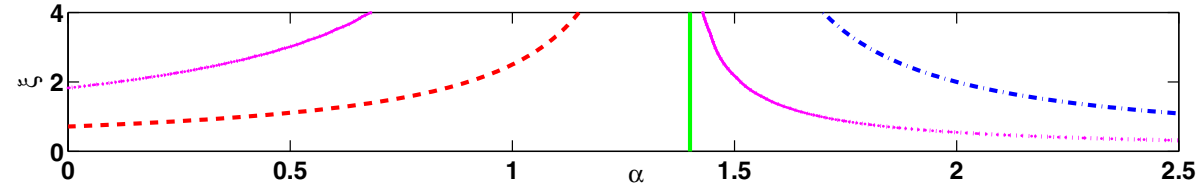

Fig. 3 Diagram for curves (10), (11), (12) under conditions (7), (8), (9) when $\beta=0.35, \delta=0.25, \theta=4$. Dashed line represents curve equation (10), dash dotted line represents curve equation (11), dotted line represents curve equation (12) and continuous line represents the straight line $\alpha=\frac{\beta}{\delta}$. a for $\gamma=2.1$ [following condition (7) (for fixed $\alpha$ and increasing value of $\xi$ in between 2 magenta colored curve branch system is stable when values of $\xi$ lies over the upper branch )], b for $\gamma=4.2$ [following condition (8) (for fixed $\alpha$ and increasing

$1+\alpha \xi+x^{*}=\frac{y^{*} \gamma^{\theta}}{\gamma^{\theta}-\left(x^{*}\right)^{\theta}}=\frac{\beta\left(x^{*}+\xi\right)}{\delta}$.

Solving the above equations we obtain

$$
\xi=\frac{\gamma^{\theta} y^{*} \delta}{\left(\gamma^{\theta}-\left(x^{*}\right)^{\theta}\right) \beta}-x^{*}
$$

value of $\xi$ in between 2 magenta colored curve branch system is stable when values of $\xi$ lies over the upper branch )], $\mathbf{c}$ for $\gamma=7.88$ [following condition (9) (for fixed $\alpha$ and increasing value of $\xi$ in between 2 magenta colored curve branch system is stable when values of $\xi$ lies over the upper branch when $\alpha<1.4$ and for $\alpha>1.4$ system is stable when $\xi$ lies under the magenta colored curve branch )] respectively are plotted in $(\alpha, \xi)$ space

$$
\text { and } \alpha=\frac{\left[\beta\left(x^{*}+\xi\right)-\left(1+x^{*}\right) \delta\right]}{\delta \xi} \text {. }
$$

\section{Global stability}

The interior equilibrium point $\left(x^{*}, y^{*}\right)$ will be globally asymptotically stable if 
$\frac{\beta\left(M^{2}-y^{*} \xi\right)}{1+\alpha \xi+M}+y^{*} \delta<x^{*}-\left(\frac{\gamma^{\theta} M^{\frac{1-\theta}{2}}+x^{\theta} M^{\frac{\theta-1}{2}}}{2}\right)^{2} \frac{1}{\gamma^{\theta}}$

(assuming $\left(x^{*}, y^{*}\right)$ is locally asymptotically stable).

Proof Let us consider the following Lyapunov function

$$
\begin{aligned}
V(x, y)= & \left(x-x^{*}\right) \ln \left(\frac{x}{x^{*}}\right)+\left(y-y^{*}\right) \ln \left(\frac{y}{y^{*}}\right) . \\
\text { Then } \frac{d V}{d t}= & \frac{\left(x-x^{*}\right)}{x} \frac{d x}{d t}+\frac{\left(y-y^{*}\right)}{y} \frac{d y}{d t} \\
= & \left(x-x^{*}\right)\left[1-\frac{x^{\theta}}{\gamma^{\theta}}-\frac{y}{1+\alpha \xi+x}\right] \\
& \quad+\left(y-y^{*}\right)\left[\frac{\beta(x+\xi)}{1+\alpha \xi+x}-\delta\right] \\
\leq & \left(x-x^{*}\right)\left(1-\frac{x^{\theta}}{\gamma^{\theta}}\right)+\frac{\beta x y}{1+\alpha \xi+x}-\frac{\beta \xi y^{*}}{1+\alpha \xi+x}+\delta y^{*} \\
\leq & \left(x-x^{*}\right)\left(1-\frac{x^{\theta}}{\gamma^{\theta}}\right)+\frac{\beta M^{2}}{1+\alpha \xi+M}-\frac{\beta \xi y^{*}}{1+\alpha \xi+M}+\delta y^{*} .
\end{aligned}
$$

Here $M=\frac{2}{\lambda}$ and $\lambda=\min \{1, \delta\}$. After simplification we get,

$$
\begin{aligned}
\frac{d V}{d t} \leq & \frac{-1}{\gamma^{\theta}}\left[x^{\frac{\theta+1}{2}}-\frac{\gamma^{\theta} x^{\frac{1-\theta}{2}}+x^{*} x^{\frac{\theta-1}{2}}}{2}\right]^{2}-x^{*} \\
& +\left[\frac{\gamma^{\theta} x^{\frac{1-\theta}{2}}+x^{*} x^{\frac{\theta-1}{2}}}{2}\right]^{2} \frac{1}{\gamma^{\theta}} \\
& +\frac{\beta M^{2}}{1+\alpha \xi+M}-\frac{\beta \xi y^{*}}{1+\alpha \xi+M}+\delta y^{*} \\
\leq & \frac{-1}{\gamma^{\theta}}\left[x^{\frac{\theta+1}{2}}-\frac{\gamma^{\theta} x^{\frac{1-\theta}{2}}+x^{*} x^{\frac{\theta-1}{2}}}{2}\right]^{2}-x^{*} \\
& +\left[\frac{\gamma^{\theta} M^{\frac{1-\theta}{2}}+x^{*} M^{\frac{\theta-1}{2}}}{2}\right]^{2} \frac{1}{\gamma^{\theta}} \\
& +\frac{\beta M^{2}}{1+\alpha \xi+M}-\frac{\beta \xi y^{*}}{1+\alpha \xi+M}+\delta y^{*} .
\end{aligned}
$$

Hence system (4) will be globally asymptotically stable if $-x^{*}+\left(\frac{\gamma^{\theta} M^{\frac{1-\theta}{2}}+x^{*} M^{\frac{\theta-1}{2}}}{2}\right)^{2} \frac{1}{\gamma^{\theta}}+\frac{\beta\left(M^{2}-y^{*} \xi\right)}{1+\alpha \xi+M}+y^{*} \delta<0$ i.e. $\frac{\beta\left(M^{2}-y^{*} \xi\right)}{1+\alpha \xi+M}+y^{*} \delta<x^{*}-\left(\frac{\gamma^{\theta} M^{\frac{1-\theta}{2}}+x^{*} M^{\frac{\theta-1}{2}}}{2}\right)^{2} \frac{1}{\gamma^{\theta}}$.

\section{Simulation results}

We represent the characteristics of system (4) according to the condition of stability and existence of interior equilibrium when $\xi=0$ by the help of numerical simulation. We take values of ecosystem parameters as $\beta=.35$, $\delta=.25, \gamma=5.5$. These values satisfies condition (9) which indicates existence of stable limit cycle for the system having $\xi=0$ about the points $(\tilde{x}, \tilde{y})=(2.5,2.7769)$, $(\tilde{x}, \tilde{y})=(2.5,3.1713),(\tilde{x}, \tilde{y})=(2.5,3.3506)$ for $\theta=2, \theta=$ 3 and $\theta=4$ respectively. As $\theta=1$ is studied by Srinivasu (cf. Srinivasu et al. 2007) we emphasis on values of $\theta$ rather than $\theta=1$. Here $\frac{\beta}{\delta}=1.400$. At first we examine the system (4) for $\theta=1,2,3,4$ by taking $(\alpha, \xi)=(0,0)$. Then we see from Fig. 4 that the system is stable only at $\theta=1$ and for other values of $\theta$ system is unstable. Therefore the variation of intraspecific competition has significant impacts over the stability of an ecosystem.

Now we fix the target prey and predator to be $(2.5,5)$. From the stability conditions [from (15 to 16)] of the fixed point $(2.5,5)$ for $\theta=2$ we get the values of $(\alpha, \xi)=(1.400,4.5015)$. Since the value of $g^{\prime}\left(x^{*}\right)=$ -0.8268 which indicates that system is stable. Now fixing the target prey and predator to be $(3.5,6)$ for $\theta=3$ then from (15) and (16) we get $(\alpha, \xi)=(1.4693,5.7736)$ and value of $g^{\prime}\left(x^{*}\right)=-2.1255$ which indicates that system is stable. Next we fix the target prey and predator to be $(3.5,6)$ for $\theta=4$ then from (15) and (16) we get $(\alpha, \xi)=$
Fig. 4 Diagram representing global stability criteria

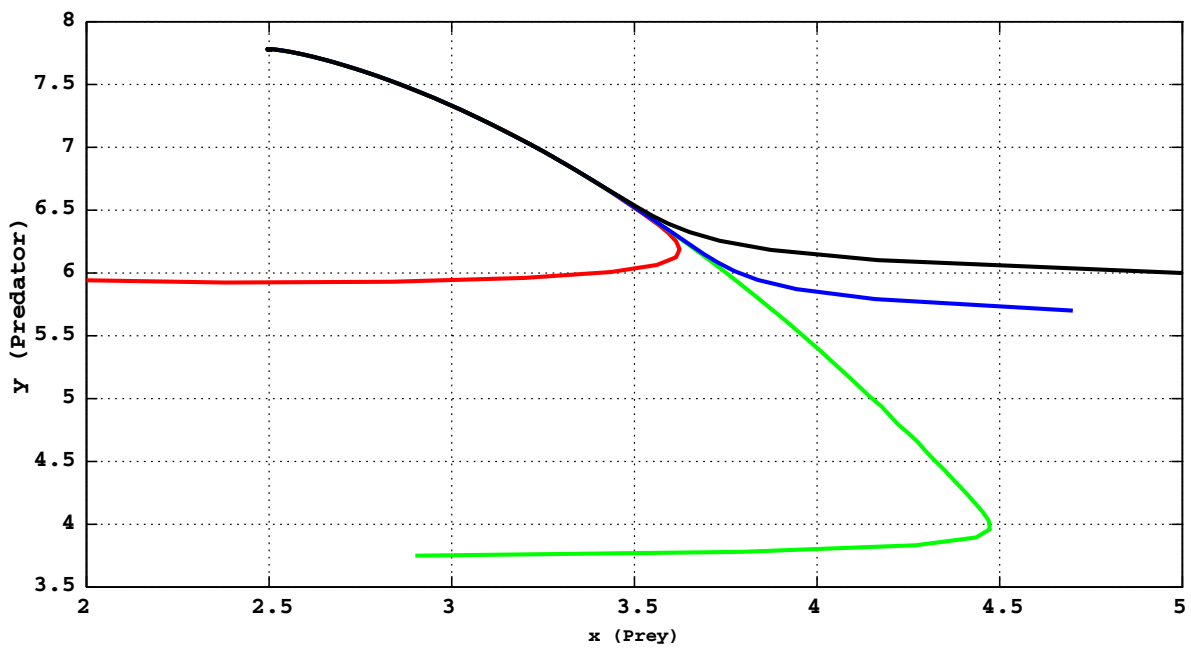


Fig. 5 Time evolution of the system (4) without additional food when $\beta=0.35, \delta=$ $0.25, \gamma=5.5$ and $\mathbf{a}$ for $\theta=1$, $\mathbf{b}$ for $\theta=2$, c for $\theta=3$ (d) for $\theta=4$ are plotted. Dashed line represents the prey population and continuous line represents the predator population (a)

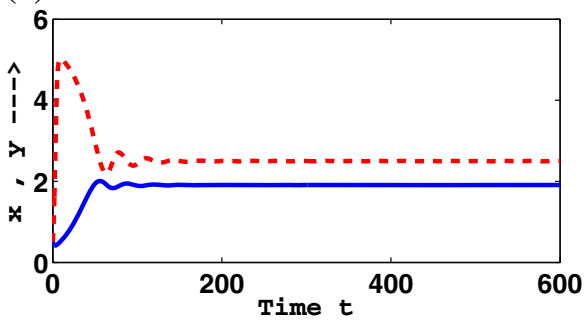

(c)

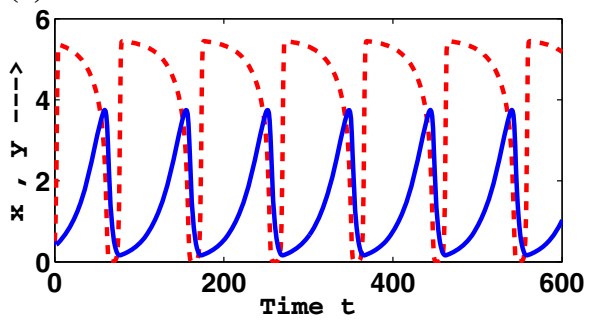

(b)

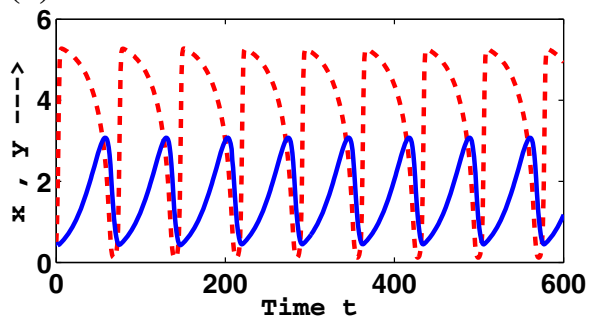

(d)

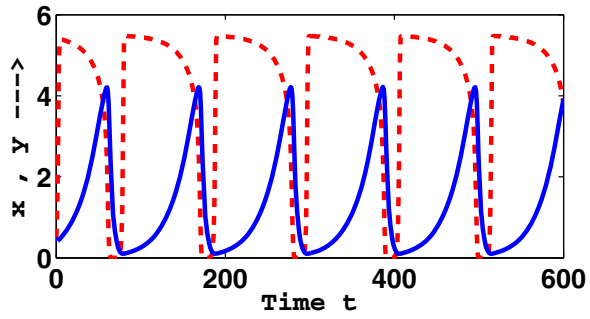

(a)

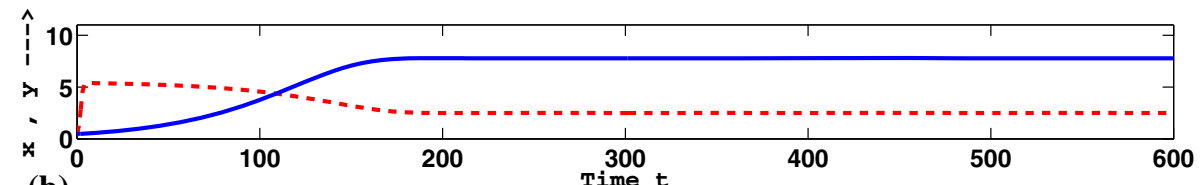

(b)

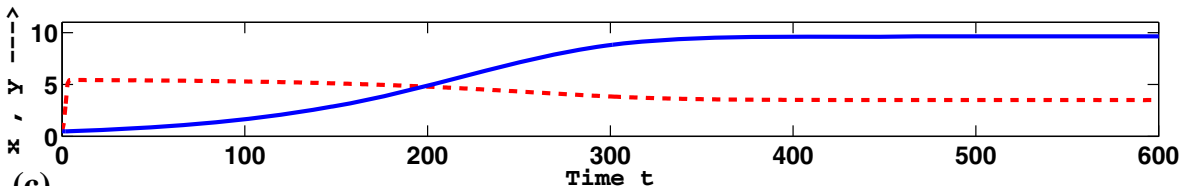

(c)

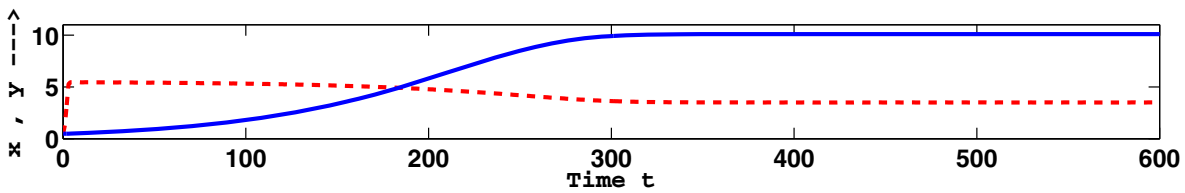

Fig. 6 Time evolution of the system (4) with $\beta=0.35, \delta=0.25, \gamma=$ 5.5 are plotted for different targeted prey predator values. a for $\theta=2, x=2.5, y=5, \alpha=1.400, \xi=4.5015$. b for $\theta=3, x=3.5$,

$(1.44780,5.1264)$ and value of $g^{\prime}\left(x^{*}\right)=-1.4274$ which indicates that system is stable. This phenomenon is depicted by Fig. 6.

Again for $\theta=2$ we fix the target prey and predator to be $(1.5,5)$ for then $(\alpha, \xi)=(1.2963,3.8584)$ and value of $g^{\prime}\left(x^{*}\right)=0.1817$ which indicates that system is unstable. Here $\alpha=1.2963<\frac{\beta}{\delta}$. Keeping $\xi=3.8584$ and target prey and predator fixed when we take $\alpha=1.43$ then system becomes stable, here bifurcation occurs with respect to $\alpha$ and time series representation is illustrated by Fig. 7 .

Again when we fixed $\theta=3$ and fix the target prey and predator $(3.5,6)$, then $(\alpha, \xi)=(1.4693,5.7736)$. Keeping $\xi=5.7736$ and target prey and predator fixed when we take $\alpha=1.35$ then system becomes unstable, here $y=6, \alpha=1.4693, \xi=5.7736$. $\quad$ c for $\quad \theta=4, x=3.5, y=6, \alpha=$ 1.4780, $\xi=5.1264$. Dashed line represents the prey population and continuous line represents the predator population

bifurcation occurs with respect to $\alpha$ hence time evolution is illustrated by Fig. 8 .

Again when we fixed $\theta=4$ and fix the target prey and predator $(3.5,8)$, then $(\alpha, \xi)=(1.4585,6.8352)$. Keeping $\xi=6.8352$ and target prey and predator fixed when we take $\alpha=1.39$ then system becomes unstable, here bifurcation occurs with respect to $\alpha$ and Fig. 9 illustrates the time evolution.

Now for $\theta=2$, we keep $\alpha=\frac{\beta}{\delta}=1.400$ fixed, then from (13) we get $\xi=0.9296$ which is the critical value of $\xi$ for Hopf bifurcation. When we take $\xi=3.59>0.9286$ then system remains stable, and when $\xi=0.86<0.9286$ system becomes unstable. Fig. 10 shows the time evolution diagram.

Next for $\theta=3$, we keep $\alpha=\frac{\beta}{\delta}=1.400$ fixed, then from (13) we get $\xi=3.2429$ which is the critical value of $\xi$ for 
Fig. 7 Time evolution of the system (4) with $\theta=2$ and $\beta=$ $0.35, \delta=0.25, \gamma=5.5$,

$\xi=3.8584$, and $x=1.5, y=5$ are plotted. a for $\alpha=1.43$. $\mathbf{b}$ for $\alpha=1.2963$. Dashed line represents the prey population and continuous line represents the predator population (a)

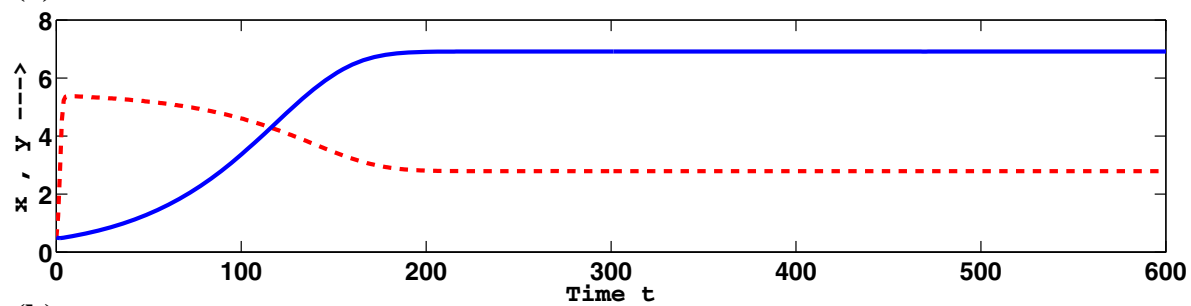

(b)

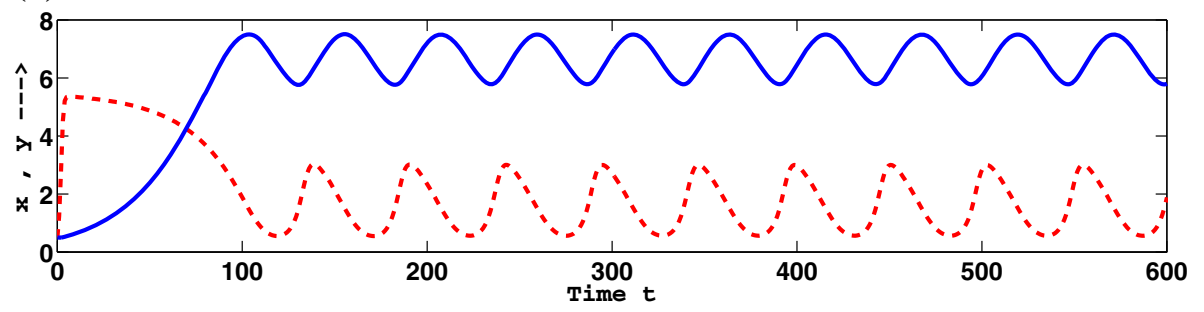

(a)

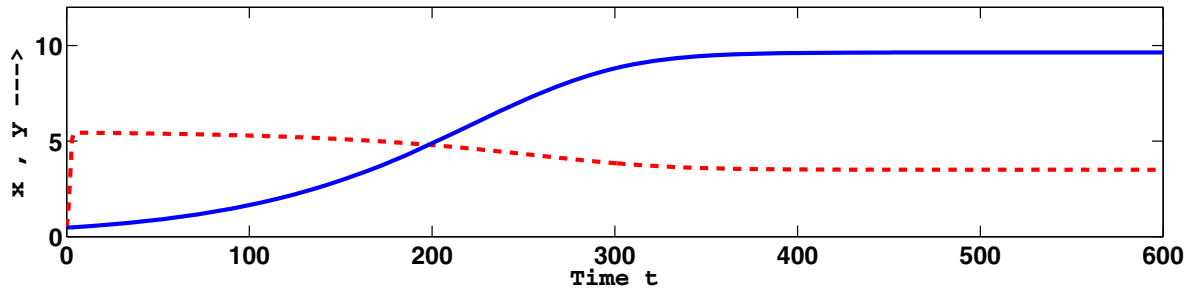

(b)
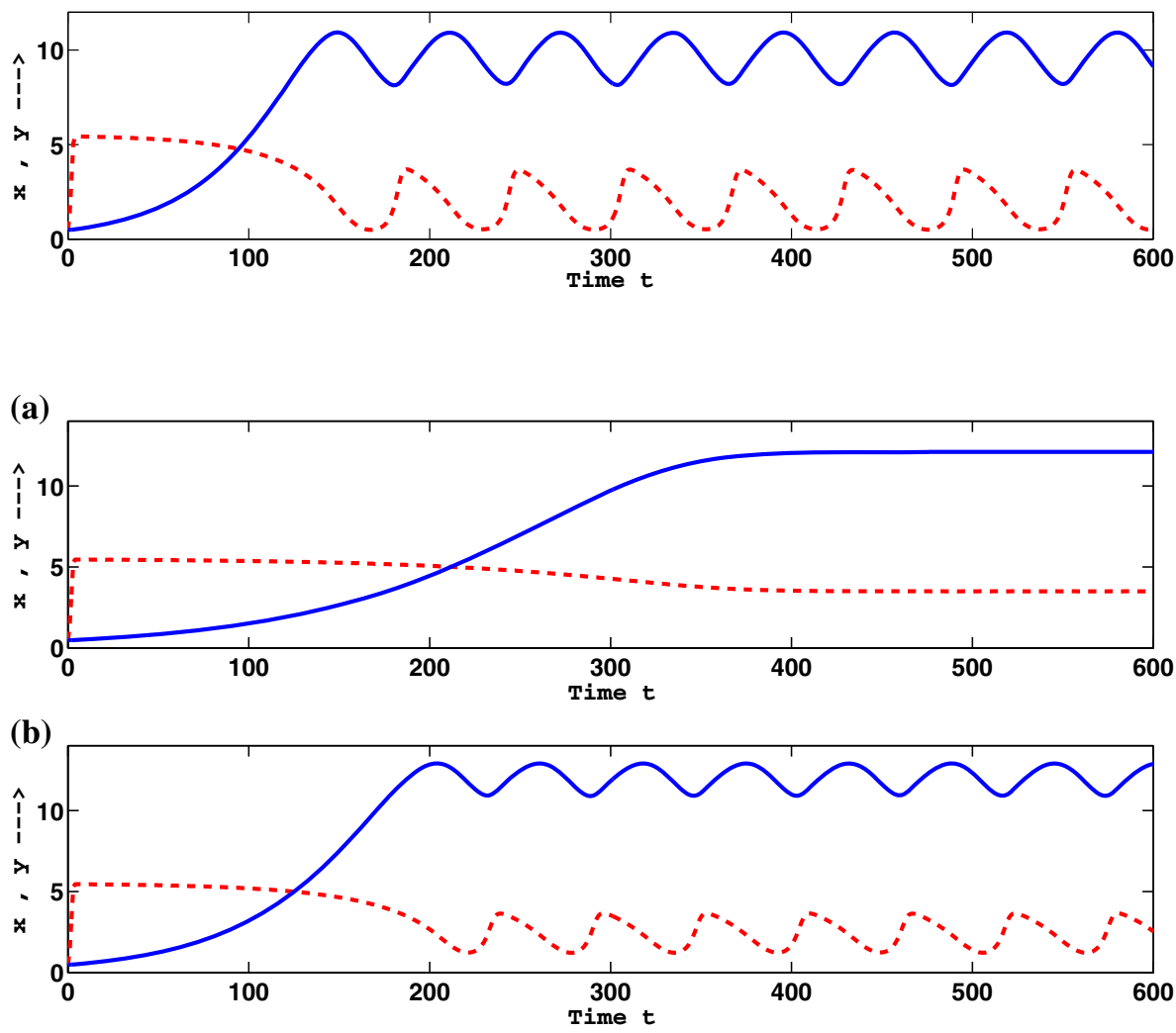

Fig. 9 Time evolution of the system (4) with $\theta=4$ and $\beta=$ $0.35, \delta=0.25, \gamma=5.5, \xi=$ 6.8352 and $x=3.5, y=8$ are plotted. a for $\alpha=1.4585$. $\mathbf{b}$ for $\alpha=1.39$. Dashed line represents the prey population and continuous line represents the predator population
Fig. 8 Time evolution of the
system (4) with $\theta=3$ and $\beta=$ $0.35, \delta=0.25, \gamma=5.5, \xi=$ 5.7736 and $x=3.5, y=6$ are $\alpha=1.35$. Dashed line represents the prey population and continuous line represents the predator population 
Fig. 10 Time evolution of the system (4) with $\theta=2$ and $\beta=$ $0.35, \delta=0.25, \gamma=5.5$ are plotted. a for $\xi=3.59$. b for $\xi=0.86$. Dashed line represents the prey population and continuous line represents the predator population (a)

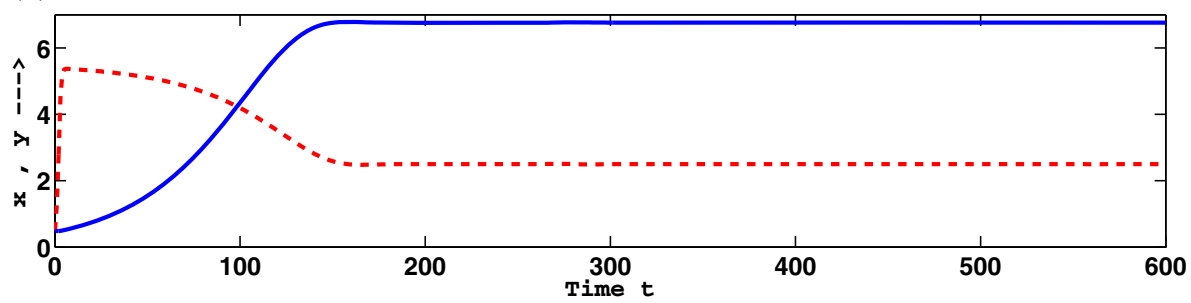

(b)

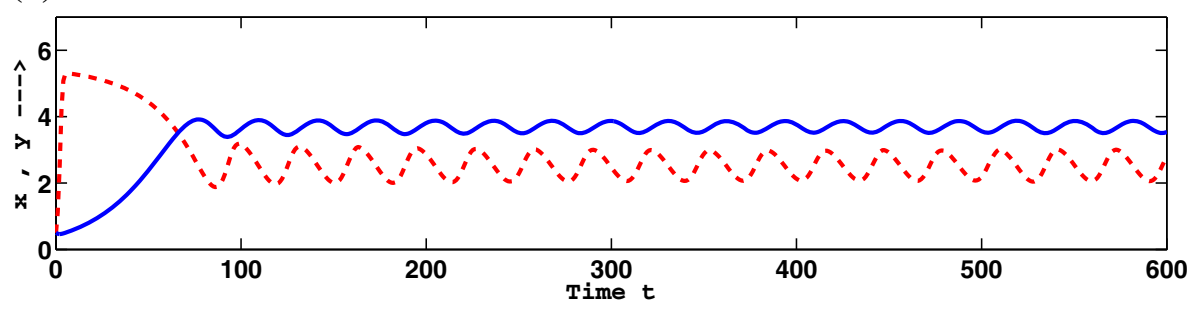

(a)

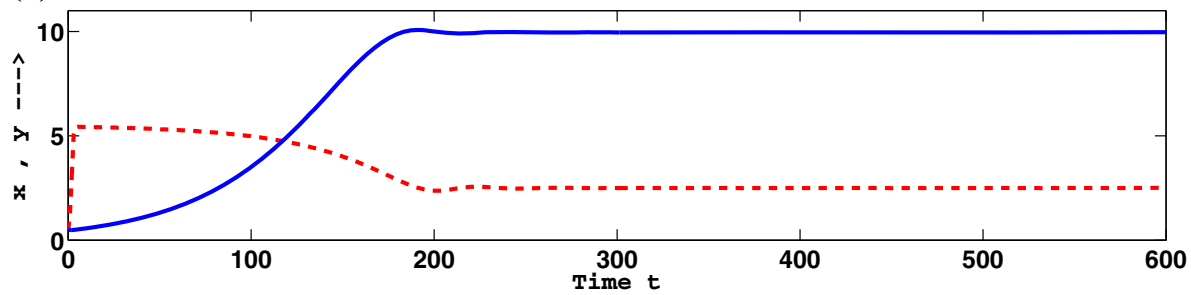

(b)

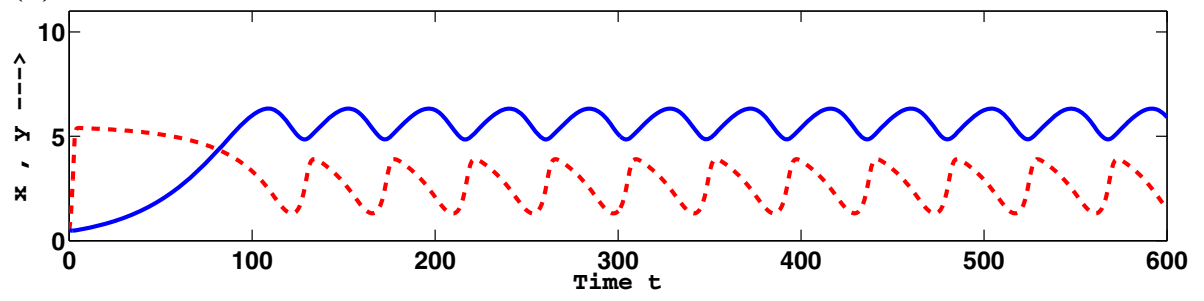

Fig. 11 Time evolution of the system (4) with $\theta=3$ and $\beta=$ $0.35, \delta=0.25, \gamma=5.5$ are plotted. a for $\xi=5.35$. b for $\xi=2.25$. Dashed line represents the prey population and continuous line represents the predator population quantity of additional food for the stability of system (4). When $\theta=2$,if $\alpha=1.2963<\frac{\beta}{\delta}=1.400$ when $\xi=3.8584$ then the system becomes unstable, and when $\alpha=1.43>\frac{\beta}{\delta}=$ 1.400 system remains stable. When $\theta=3$, if $\alpha=1.35<\frac{\beta}{\delta}=$ 1.400 when $\xi=5.7736$ then the system is unstable, and when $\alpha=1.469>\frac{\beta}{\delta}=1.400$ system becomes stable. When $\theta=4$, if $\alpha=1.39<\frac{\beta}{\delta}=1.400$ when $\xi=6.8352$ then the system becomes unstable, and when $\alpha=1.4585>\frac{\beta}{\delta}=$ 1.400 system remains stable. From the above data it is clear that when $\alpha<\frac{\beta}{\delta}$ for any value of $\theta=2,3,4$ system becomes unstable and when $\alpha>\frac{\beta}{\delta}$ then the system remains stable. When $\alpha=\frac{\beta}{\delta}$ then the system goes under Hopf bifurcation with respect to $\xi$ for various values of $\theta$. The following figure represents bifurcation diagram of predator population with respect to $\xi$ for various values of $\theta$. 
Fig. 12 Time evolution of the system (4) with $\theta=4$ and $\beta=$ $0.35, \delta=0.25, \gamma=5.5$ predator-prey system are plotted. a for $\xi=9.9$, b for $\xi=6.9$. Dashed line represents the prey population and continuous line represents the predator population

(a)

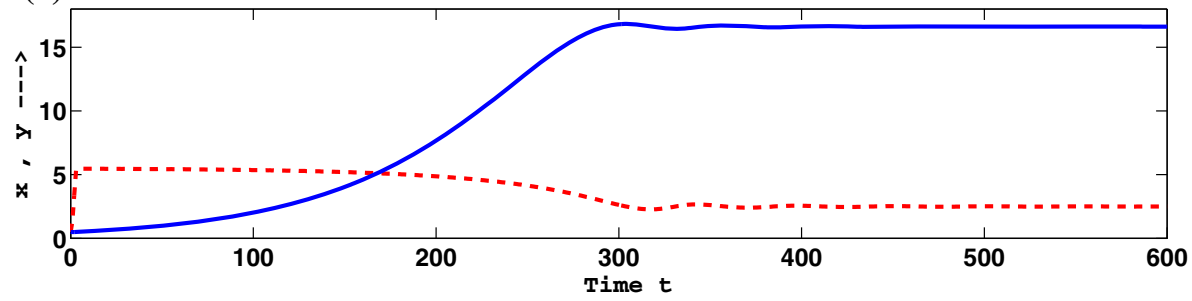

(b)
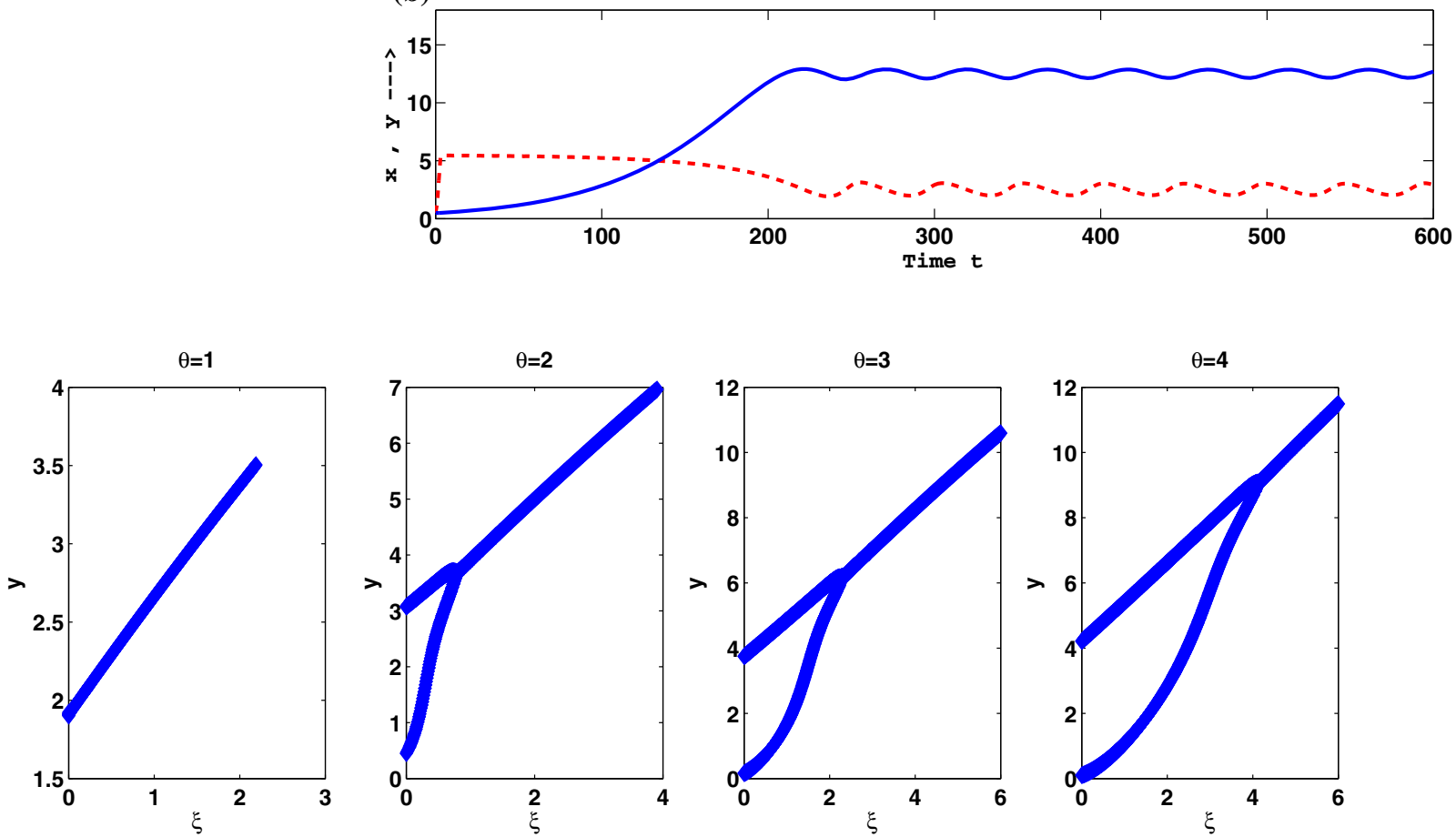

Fig. 13 Bifurcation Diagrams of the system (4) with respect to $\xi$ are plotted. a for $\theta=1, \mathbf{b}$ for $\theta=2, \mathbf{c}$ for $\theta=3$, $\mathbf{d}$ for $\theta=4$

\section{Conclusions}

Systematic analysis of the dynamics of prey predator system with additional food for predator along with intraspecific competition among prey population is done in this paper. In nature competition for food among carrion feeders and fruit flies is observed (cf. Klomp 1964). Beetles are seen to compete for resource cricket eggs (cf. Griffith and Poulson 1993), wood butterfly (cf. Gibbsac et al. 2004) also used to take part in intraspecific competition. The intraspecific competition among prey is incorporated in the model through the theta-logistic growth rate of prey. The local and global stability analysis of the model are done. Conditions for Hopf bifurcation are derived analytically and verified numerically. It is observed that intraspecific competition between prey plays a vital role in stability and existence criteria of the interior equilibrium point. Moreover, it is found that in absence of additional food the interior fixed point of the system become unstable with the increase of intraspecific competition among prey. Therefore prey will extinct when the intraspecific competition crosses a critical value. Here additional food is characterized by to be of high quality if $\alpha<\frac{\beta}{\delta}$ and is of low quality when $\alpha>\frac{\beta}{\delta}$.

For fixed additional food the increase of intraspecific competition can make the system (4) unstable which indicates that predator may extinct as a result of extinction of prey. Coexistence of predator and prey is possible only when we increase the amount of additional food suitable with the increase of intraspecific competition. Hence, alternative food can control the dynamics of the ecosystem in presence of intraspecific competition also. Therefore, all most all results of Srinivasu (cf. Srinivasu et al. 2007) will be also valid in presence of intraspecific competition among prey. This investigation generalizes the existing knowledge of the effects of additional food in a food chain model. Our theoretical results may be useful to analyse some experimental data set of prey-predator system. The 
effects of additional food on the dynamics of a food chain model in presence of intraspecific competition in both prey and predator species may be an area for future study to model real world ecological systems.

\section{References}

Agiza HN, Elabbasy EM, El Metwally H, Elsadany AA (2009) Chatoic dynamics of a discrete prey-predator model with Holling type II. Non Linear Anal Real World Appl 10:116-129

Manju Agarwal, Rachana Pathak (2012) Harvesting and hopf bifurcation in a prey-predator model with Holling type IV functional response. Int J Math soft comput 2(1):83-92

Akcakaya H, Arditi R, Ginzburg L (1995) Ratio-dependent predation: an abstraction that works. Ecology 76(3):995

Aziz-Alaoui M, Okiye MD (2003) Boundedness and global stability for a predator-prey model with modified LeslieGower and Holling-type II schemes. Appl Math Lett 16:1069-1075

Camara BI, Aziz - Alaoui MA, (2008) Complexity in a prey predator model. Int Conf Honor Claude Lobry 9:109-122

Chakraborty S, Tiwari PK, Misra AK, Chattopadhyay J (2015) Spatial dynamics of a nutrient phytoplankton system with toxic effect on phytoplankton. Math Biosci 264:94-100

Gakkhar S, Singh A (2012) Control of chaos due to additional predator in the Hastings Powell food chain model. J Math Anal Appl 385:423-438

Gibbsac M, Lacea LA, Jonesa MJ, Mooreb AJ (2004) Intraspecific competition in the speckled wood butterfly Pararge aegeria: effect of rearing density and gender on larval life history. J Insect Sci 4;(16):1-6. doi:10.1673/031.004.1601

Griffith DM, Poulson TL (1993) Mechanisms and consequences of intraspecific competition in a carabid cave beetle. Ecology 74(5): 1373-1383

Holling CS (1959a) The components of predation as revealed by a study of Small mammal predation of the European pine sawfly. Can Entomol 91:293-320

Holling CS (1959b) Some characteristics of simple types of predation and Parasitism. Can Entomol 91:385-398

Holling CS (1965) The functional response of predators to prey density and its role in mimicry and population regulation. Mem Entomol Soc Can 45:5-60

Klomp H (1964) Intraspecific competition and the regulation of insect numbers. Ann Rev Entomol 9:17-40

Kumar R, Freedman HI (1989) A mathematical model of facultative mutualism with populations interaction in a food chain. Math Biosci 97:235-261

Lu Hongying, Wang Weigno (2011) Dynamics of a delayed discrete semi-ratio dependent predator-prey system with Holling type IV functional response. Adv Diff Equ :3-19

Mukherjee Debasis, Das Prasenjit and Kesh Dipak (2011) Dynamics of a plant - herbivore model with Holling type II functional response. Comput Math Biol. Issue :2(1)

Pal S, Chatterjee S, Das KP, Chattopadhyay J (2009) Role of competition in phytoplankton population for the occurrence and control of plankton bloom in the presence of environmental fluctuations. Ecol Model 220(2):96-110

Pitchford J, Brindley J (1998) Intratrophic predation in simple predator-prey models. Bull Math Biol 60:937-953
Prasad B, Banerjee M, Srinivasu PDN (2013) Dynamics of additional food provided predator prey system with mutually interfering predators. Math Biosci 246(1):176-190

Ross JV (2009) A note on density dependence in population models. Ecol Model 220:3472-3474

Ruan S, Xiao D (2001) Global analysis in a predator-prey system with non monotonic functional response. SIAM J Appl Math 61(4):1445-1472

Sahoo B, Poria S (2011) Dynamics of a predator-prey system with seasonal effects on additional food. Int $\mathrm{J}$ Ecosyst 1:10-13

Sahoo B, Poria S (2013) Disease control in a food chain model supplying alternative food. Appl Math Model 37(8):5653-5663

Sahoo B, Poria S (2013) Oscillatory coexistence of species in a food chain model with general Holling interactions. Differ Equ Dyn Syst. doi:10.1007/s12591-013-0171-9

Sahoo B, Poria S (2013) Disease control in a food chain model supplying alternative food. Appl Math Model 37:5653-5663

Sahoo B, Poria S (2014) Effects of supplying alternative food in a predator-prey model with harvesting. Appl Math Comput 234:150-166

Sahoo B, Poria S (2014) The chaos and control of a food chain model supplying additional food to top-predator. Chaos Solitons Fractals 58:52-64

Sahoo B, Poria S (2015) Effects of allochthonous inputs in the control of infectious disease of prey. Chaos Solitons Fractals 75:1-19

Saeez E, Gonzalez-Olivares E (1999) Dynamics of a predator-prey system. SIAM J Appl Math 59(5):1867-1878

Shanshan Chen, Junping Shi, Junjie Wei (2013) The effect of delay on a diffusive predator-prey system with Holling type-II predator functional response. Commun Pure Appl Anal 12(1):481-501

Skalski GT, Gilliam JF (2001) Functional responses with predator interference: viable alternatives to the Holling type II mode. Ecology 82:3083-3092

Sabelis MW, Rijn PC (2006) When does alternative food promote biological pest control? IOBC WPRS Bull 29:428-37

Sabelis MW, van Rijn PCJ (2005) When does alternative food promote biological pest control? In: Hoddle MS (ed) Proc. Second Int. Symp. Biol, Control of Arthropods, p 428-437

Srinivasu PDN, Prasad BSRV, Venkatesulu M (2007) Biological control through provision of additional food to predators : atheoretical study. Teor Popul Bio 1(72):111-120

Srinivasu PDN, Prasad BSRV (2010) Time optimal control of an additional food provided predator prey system with applications to pest management and biological conservation. J Math Biol 60:591-613

Srinivasu PDN, Prasad B (2011) Role of quantity of additional food to predators as a control in predator prey systems with relevance to pest management and biological conservation. Bull Math Biol 73(10):2249-2276

Wang W, Wang H, Li Z (2007) The dynamic complexity of a threespecies Beddington-type food chain with impulsive control strategy. Chaos Solitons Fractals 32:1772-1785

Yujing Gao (2013) Dynamics of ratio-dependent predator prey system with a strong Allee effect. Discrete Contin Dyn Syst ser B 18(9):2283-2313

Zhang ZZ, Yang HZ, (2013) Hopf bifurcation in a delayed predatorprey system with modified Leslie-gower and Holling type III schemes. ACTA AUTOMATICA SINICA 39(5):610-616

Zhang Lei, Wang Weiming, Xue Yakui, Jin Zhen (2008) Complex dynamics of a Holling-type IV predator prey model. arXiv:0801. 4365 [q-bio.PE]:1-23 